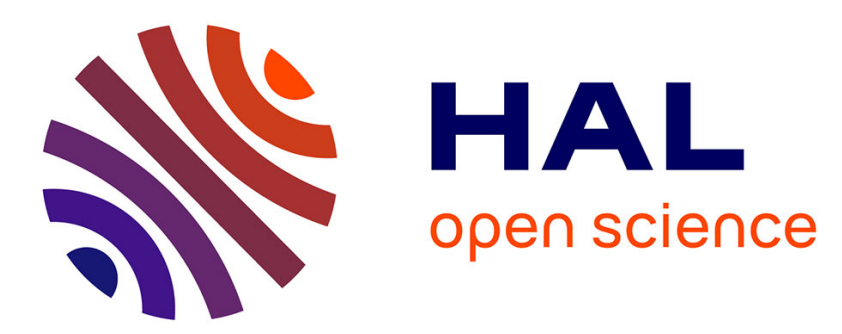

\title{
A versatile technology for colloidal crystal transfer using parylene coatings and hydrosoluble polymers
}

Andrei A Ushkov, Olivier Dellea, Olivier Lebaigue, Olivier Poncelet, Isabelle Verrier, Yaya Lefkir, Yves Jourlin

\section{- To cite this version:}

Andrei A Ushkov, Olivier Dellea, Olivier Lebaigue, Olivier Poncelet, Isabelle Verrier, et al.. A versatile technology for colloidal crystal transfer using parylene coatings and hydrosoluble polymers. Nanotechnology, 2022, 33 (18), pp.185301. 10.1088/1361-6528/ac4dc3 . hal-03562247

\section{HAL Id: hal-03562247 \\ https://hal.science/hal-03562247}

Submitted on 8 Feb 2022

HAL is a multi-disciplinary open access archive for the deposit and dissemination of scientific research documents, whether they are published or not. The documents may come from teaching and research institutions in France or abroad, or from public or private research centers.
L'archive ouverte pluridisciplinaire HAL, est destinée au dépôt et à la diffusion de documents scientifiques de niveau recherche, publiés ou non, émanant des établissements d'enseignement et de recherche français ou étrangers, des laboratoires publics ou privés. 


\title{
A versatile technology for colloidal crystal transfer using parylene coatings and hydrosoluble polymers
}

\author{
Andrei A. Ushkov ${ }^{1,2}$, Olivier Dellea ${ }^{3}$, Olivier Lebaigue ${ }^{3}$, Olivier \\ Poncelet $^{3}$, Isabelle Verrier ${ }^{1}$, Yaya Lefkir ${ }^{1}$ and Yves Jourlin ${ }^{1}$ \\ ${ }^{1}$ Laboratoire Hubert Curien UMR 5516, F-42023, Université de Lyon, \\ UJM-Saint-Etienne, CNRS, Institut d'Optique Graduate School, 18 Rue Du Pr. \\ Benoit Lauras, 42000, Saint-Etienne, France \\ ${ }^{2}$ Center for Photonics and 2D Materials, Moscow Institute of Physics and \\ Technology, 9 Institutsky Lane, 141700, Dolgoprudnyy, Russia \\ ${ }^{3}$ CEA-Liten, Grenoble Alpes Univ, 17 rue des Martyrs, 38054, Grenoble, France \\ E-mail: andrei.ushkov@univ-st-etienne.fr
}

December 2021

\begin{abstract}
We propose a novel versatile colloidal crystal transfer technique compatible with a wide range of substrates regardless of their size, material, and wettability. There are no inherent limitations of colloidal particles material and size. The method possibilities are demonstrated via the colloidal transfer on quartz, glass substrates with a flat and curved surface, and the fabrication of 3D colloidal structure with 5 overlaid colloidal monolayers. The process occurs at a room temperature in water and is independent from the illumination conditions, which makes it ideal for experimental manipulations with sensitive functional substrates. We performed the nanosphere photolithography process on a photosensitive substrate with a transferred colloidal monolayer. The metallized hexagonal arrays of nanopores demonstrated a clear resonant plasmonic behavior. We believe that due to its high integration possibilities the proposed transfer technique will find applications in large-area surface nanotexturing, plasmonics, and will speed up a device fabrication process.
\end{abstract}

\section{Introduction}

Colloidal nanoparticles, arranged into hexagonal close-packed structures and often referred as colloidal crystals, have been a subject of intense research for decades due to their capabilities for light-matter interactions [1]. The interest is caused by their prospective use in a photonic crystal regime [2], Purcell factor enhancement [3], structure color generation $[4,5]$ inspired by natural opals. Along with 3-dimensional multilayered colloidal crystals, colloidal monolayers are a flexible platform for resonant and nonresonant optical effects: moth-eye mimicking structures $[6,7,8]$, photovoltaics and light harvesting [9], Raman spectroscopy substrates [10], optical sensors [11].

The colloidal crystal deposition is an important part of device fabrication as it determines the close-packed structure quality and, consequently, its diffraction 
properties. A variety of methods have been proposed, such as spin-coating [12], dip-coating [13], electrophoretic deposition [14] and colloidal self-assembly at airliquid interface (Langmuir-Blodgett technique) [15]; they can be called "first principle" methods in analogy with first principle simulations, because they explicitly treat single nanoparticles as building blocks of the future colloidal crystal, study forces between them and substrate [16], study the influence of experimental parameters on the colloidal crystal quality $[17,18]$. Therefore, the first principle methods are important from the fundamental point of view, and allow producing high-quality colloidal crystals as well.

However, the methods introduced above usually consider a certain combination of substrate and/or nanoparticle materials, thus limiting the applicability of elaborated experimental conditions to the most common cases only, like, for example, silicon/glass substrate and silica/polystyrene nanoparticles $[14,15]$. In order to expand the applicability of self-assembled colloidal crystals, a relatively new branch of methods has been developed based on a colloidal crystal transfer from initial substrate to the final one $[19,20,21,22,23,24]$. In contrast to first principle methods, these techniques do not generally perform a colloidal self-assembly as the most sophisticated and delicate procedure, but are aimed for the transfer of already prepared colloidal crystals on device being fabricated.

Authors of [20] describe a particle transfer printing with a thin water film as an intermediate adhesion layer in a transfer process. The technology allows the selective transfer of colloidal structure to form complex 2D patterns, but is limited to silica spheres and silicon target substrates (with a thin oxide layer) only. In [23] a freestanding poly(vinyl alcohol) (PVA)-based colloidal films were proposed to use in photovoltaics, but the process simplicity was achieved at a cost of increased film thickness of 5070 microns. A big thickness might be undesirable for applications where near-field colloidal-structure-induced diffraction effects (i.e. photonic nanojets) should be located in the vicinity of the target surface. The work [21] presents an elaborated approach for layer-by-layer transfer of colloidal crystals with an arbitrary controlled number of layers. The method demonstrates a good repeatability, allows composition of layers with different morphology, particle size and materials. On the other hand, this approach is difficult to implement into device fabrication with sensitive functional films demanding to the environmental conditions like photoresists and photocatalytic surfaces, because it utilizes tough steps like heating at $100{ }^{\circ} \mathrm{C}$ for $3 \mathrm{~h}$ and PVA calcination at $550{ }^{\circ} \mathrm{C}$ for 5h. The same remark applies to the work [24] as well, where the sacrificial polymethylmethacrylate (PMMA) thin film was degraded under $254 \mathrm{~nm}$ UV irradiation for $30 \mathrm{~min}$. It should be noted that intensive UV irradiation during the transfer process is incompatible with perspective Nanosphere Photolithography (NPL) method of surface nanostructuration [25, 26], where photonic nanojets are used for resist exposure. NPL-fabricated metasurfaces are used in active photonics [27], surface texturing [28], plasmonic sensors [29] etc.

We believe that the progress in nanofabrication will definitely require the colloidal crystal depositions on non-conventional substrates with complex geometries and physical 
properties - curved, large-area hydrophilic/hydrophobic functional surfaces - which are demanding about environmental and treatment conditions. Consequently, standard selfassembly and transfer techniques are often not applicable. In this paper, we propose a novel technique of colloidal crystal transfer that is free of the constraints mentioned above. Our approach can be used for the deposition of inorganic (glass, ceramic, ceramic oxides such as $\mathrm{SiO}_{2}, \mathrm{Al}_{2} \mathrm{O}_{3}$, metallic) and organic (e.g., polystyrene) nanoparticles on any textured/non textured functional target surface regardless of its hydrophobility and curvature, with only condition of being water-insoluble; the transfer process can occur at room temperatures and doesn't utilize UV irradiation, which is exceptionally important for photosensible substrates. The proposed technology is described in detail in the patent [30]. We demonstrate the colloidal crystal transfer on target substrates made of glass and quartz, flat and curved, with diameter of nanoparticles of $580 \mathrm{~nm}, 1.1 \mu \mathrm{m}$ and $2.5 \mu \mathrm{m}$. Moreover, by repeating transfer steps colloidal multilayered structures can be obtained, we demonstrate up to 5 deposited layers. We integrated the transfer process into NPL routine and got arrays of hexagonally arranged nanopores of adjustable depth. The quality of the overall process was confirmed by observing the plasmonic-mediated Extraordinary Optical Transmission (EOT) through metallized structures.

\section{Methods}

\section{Colloidal crystal transfer}

Here we explain the principles of the proposed colloidal crystal transfer technique. Main steps are shown in Fig.1 and demonstrate two main procedures: the preparation of largearea thin sheets of colloidal crystal monolayers encapsulated between initial substrate and cover for the ease of transportation (Figs.1a-c) and the transfer process itself on any substrate on demand (Figs.1d-f).

The close-packed colloidal monolayer is formed on the initial substrate using conventional Langmuir-Blodgett technique [1]; without loss of generality silica particles were used in our study, whereas other materials are also possible due to the proper adaptation of Langmuir-Blodgett technique and experimental conditions. The size of colloidal particles used is $580 \mathrm{~nm}, 1.1 \mu \mathrm{m}$ and $2.5 \mu \mathrm{m}$ in diameter. The initial substrates were chosen to be $160 \mu \mathrm{m}$-thick polycarbonate sheets of size of $10 \times 10 \mathrm{~cm}$, they were fully covered with close-packed colloidal monolayer. In the second step an ultra-thin (40 nm) parylene coating is deposited on the top of colloidal crystal, denoted in green in Fig. 1b. Parylene polymer coatings have good chemical barrier properties, are thermally- and UV-stable for irradiation doses used in lithography [31], which makes these coatings compatible with NPL. The parylene mechanical stability and insolubility at room temperatures mechanically protects the colloidal crystal during all subsequent manipulations. In the third step (Fig.1c) the preparation of transportable colloidalcontaining sheets is finalized by deposition of an initial $20 \mu \mathrm{m}$-thick hydrosoluble PVA cover via spin-coating of its 5 wt $\%$ aqueous solution. The resulting sheets with 


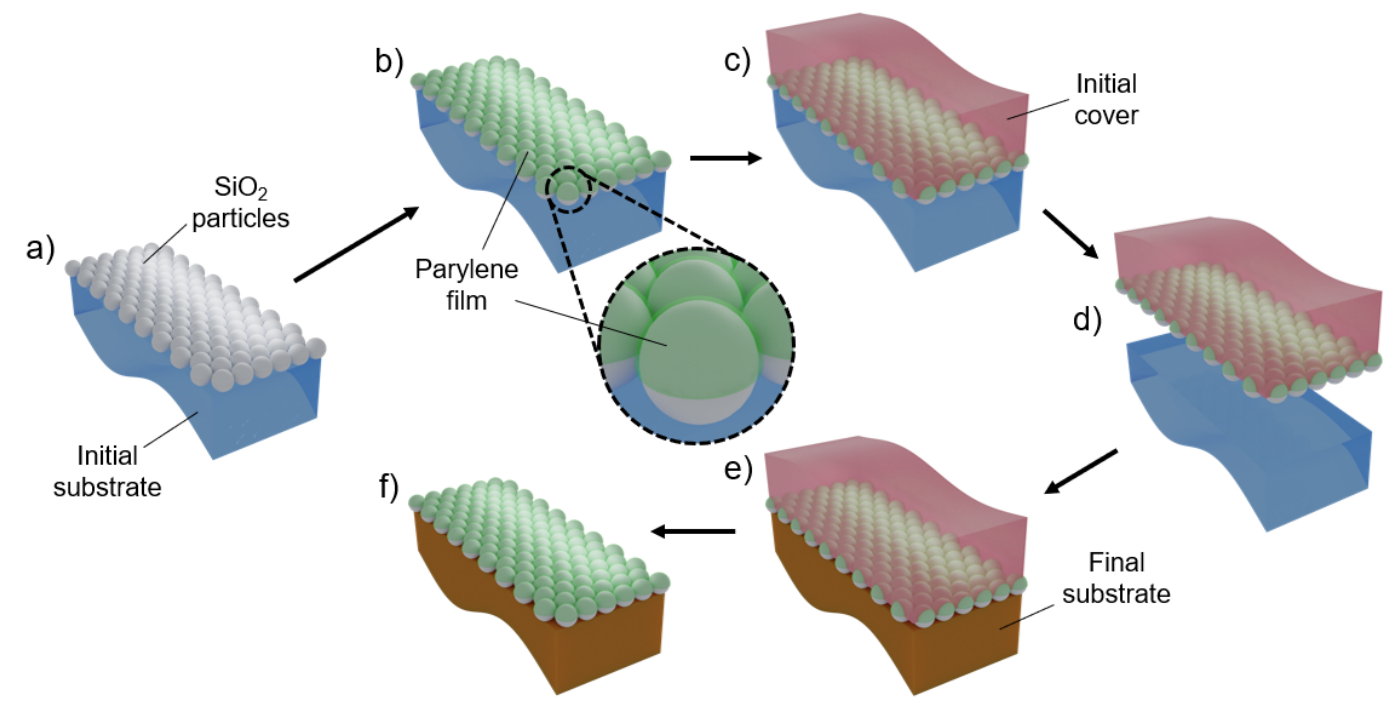

Figure 1. The scheme of proposed colloidal crystal transfer process. a) The colloidal self-assembly and deposition on an initial substrate, for example, polycarbonate; b) Thin ( 40 nm) parylene coating deposition, depicted as green transparent film; c) Deposition of an initial cover containing hydrosoluble polymer, for example, PVA; d) The colloidal monolayer and initial cover peeling off from the initial substrate; e) Contact with a final substrate; f) Dissolution of the initial cover in water.

encapsulated colloidal monolayers can be stored and transported without any special vibrational and temperature requirements, they only should be contained in standard office punched pockets to avoid a high air humidity.

The second procedure is the colloidal crystal transfer from the prepared sheets to any substrate on demand. As the adhesion of parylene-covered colloidal crystals with the initial cover made of PVA is much stronger than that with the initial polycarbonate substrate, the nanoparticles with stay on the lower side of the initial cover after a simple manual peeling (see Fig.1d and Fig.2a). The free-standing PVA-supported colloidal crystal is manually applied to the target substrate (Fig.1e and Fig.2b) with a certain pressure. As the target substrate can be of different materials, in order to increase the adhesion with it the sample is immersed in water for a short time $\sim 1$ s at room temperature, to provoke the PVA layer partial melting. Then, to delete water and harden the PVA layer again, sample is dried under a nitrogen stream, or can be placed on a hot plate at $30^{\circ} \mathrm{C}$ for delicate drying (Fig.2c). In order to complete the transfer the initial PVA cover should be eliminated. To do this, the sample from Fig.1e is immersed in a deionized (DI) water tank at room temperature for $\sim 30 \mathrm{~min}$, with subsequent water pumping out of the tank at low speed to ensure a high-quality colloidal transfer (Fig.2d). As a result, the close-packed colloidal crystal encapsulated in a polymer sheet is transferred on the target substrate (Fig.1f and Fig.2e). 

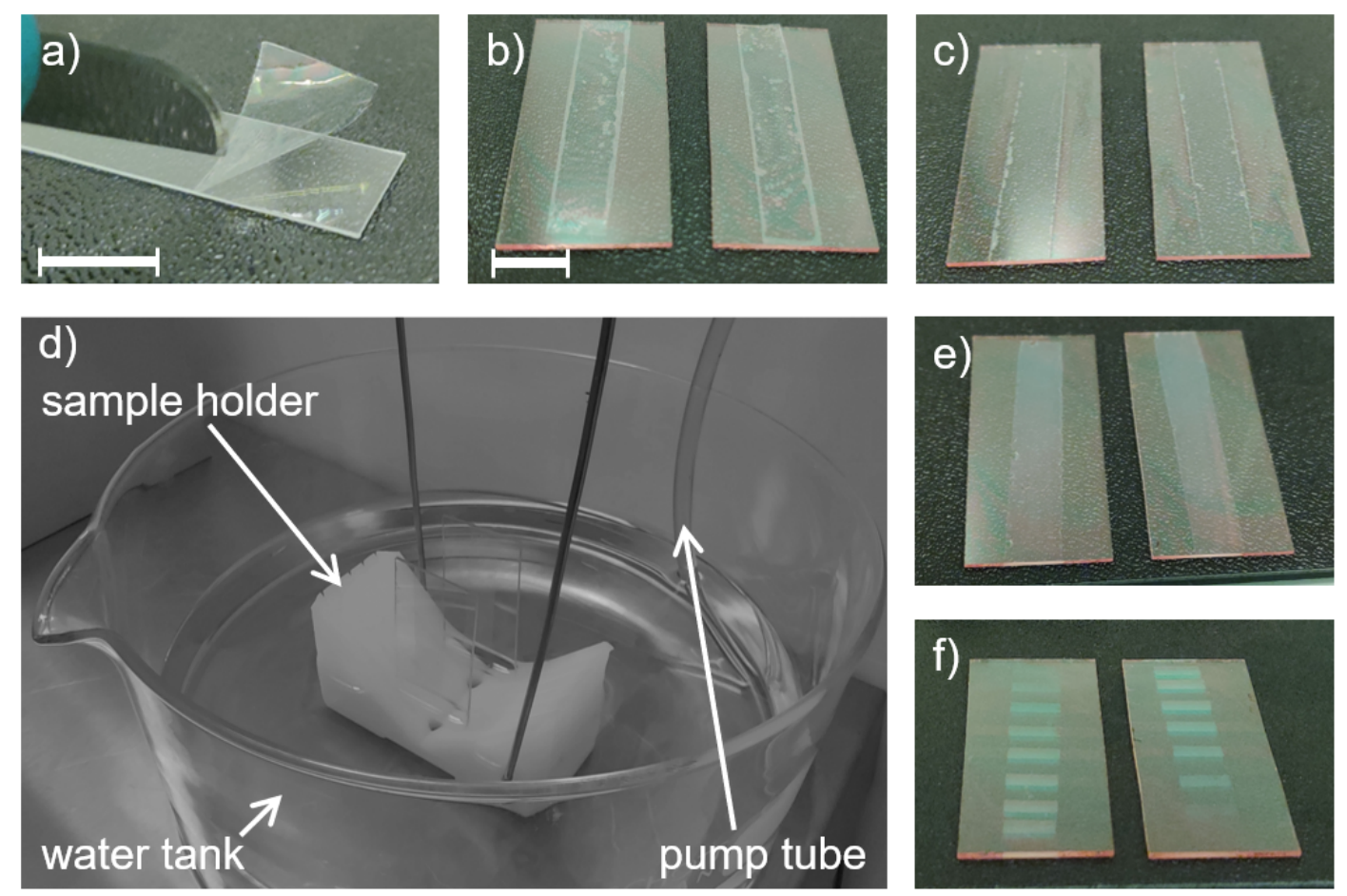

Figure 2. a)-e) Photographs of the process of colloidal crystal transfer from initial polymer sheets to the target substrate (BK7 glass slides covered with photoresist Shipley S1805 in this case): a) the peeling off the initial cover with colloidal crystal; b) the initial cover with colloidal crystal mechanically applied to the target substrate. Scale bars in a) and b) denote $1 \mathrm{~cm}$; c) samples after a short $\sim 1 \mathrm{~s}$ immersion in water for partial PVA melting, dried under the nitrogen stream; d) the process of PVA dissolution in a water tank with samples oriented vertically. Water is pumped out of the tank at low speed via the tube, so only the parylene-covered colloidal crystal stays on the substrate. f) The photograph of nanopore arrays fabricated in the photoresist via NPL, using the transferred colloidal crystal as a nanojet-forming mask. Different zones on every sample in $\mathrm{f}$ ) correspond to different UV exposure time. The diameter of transferred colloidal particles in a)-e) and the inter-pore distance in f) is $580 \mathrm{~nm}$.

\section{Nanosphere Photolithography}

The Nanosphere Photolithography (NPL) is a high-throughput, relatively inexpensive approach for large-area surface nanopatterning, based on the inhomogeneous exposure of photoresist by nanojets [25]. The principle of NPL is sketched in Fig.3. The colloidal crystal monolayer transferred on the photoresist surface is UV-exposed during the time $t_{\text {exp }}$; during the exposure every nanoparticle acts as a microlens, generates the nanojet and exposes the resist inhomogeneously [26]. We use a positive photoresist Shipley S1805 and a portable UV lamp E2107 $2 \times 6 \mathrm{~W}$ for UV irradiation at $254 \mathrm{~nm}$ (Fig.3a). It should be noted that, although the parylene film absorbs at wavelengths below $290 \mathrm{~nm}$ $[32,33]$, its thickness $(40 \mathrm{~nm})$ is not enough to affect the NPL process; practically it can lead only to a small increase of $t_{\text {exp }}$. As a substrate we used BK7 glass slides $3.7 \times 2.5 \mathrm{~cm}$, cleaned in a three-step wet-bench procedure (ultrasonic acetone bath, ethanol bath and 


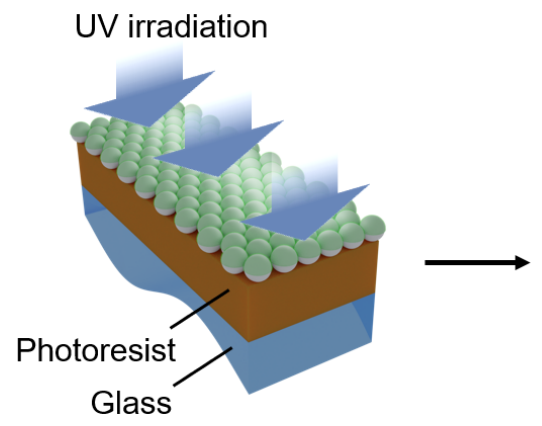

a)

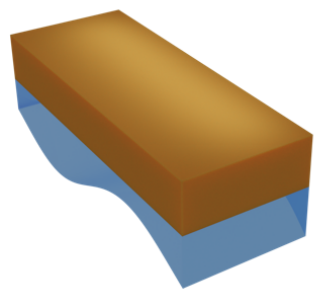

b)

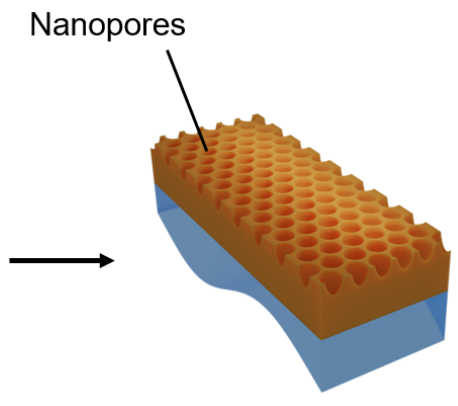

c)

Figure 3. The scheme of NPL method. a) UV-exposure of the close-packed colloidal monolayer (with a 40-nm thin parylene film denoted in green) transferred on the photoresist surface; b) The removal of colloids in an ultrasonic bath; c) The formation of hexagonal arrays of nanopores during the resist development.

deionized water bath). The 600-nm thick resist layer was spin-coated on BK7 slides and soft-baked at $60^{\circ} \mathrm{C}$ for $1 \mathrm{~min}$ on a hot plate. In the second step (Fig.3b) the colloidal mask is removed via the ultrasonic bath, and in the final step (Fig.3c) the sample is developed in MF-319 at $8{ }^{\circ} \mathrm{C}$ during the time $t_{d e v}$. The developer dissolves the resist inhomogeneously, so the arrays of nanopores appear on the surface which repeat the hexagonal arrangement of initially transferred nanoparticles. The nanopore depth depends on values of $t_{\exp }$ and $t_{d e v}$ for a given photoresist and developer.

\section{Samples characterization}

The quality of colloidal crystal transfer was characterized using scanning electron microscope Nova NanoSEM 200 FEI; the nanopore depth was measured with atomic force microscope Bruker Dimension XR; the transmission spectra were obtained via UV-Vis-NIR spectrophotometer Agilent Cary 5000.

\section{Results and discussion}

\section{Transfer on different substrates}

The colloidal transfer in Figs.2b-e and NPL process in Fig.2f performed for two samples at once demonstrates a high-throughput, repeatability and stability of the method. The colloidal crystal can be transferred on target substrates of different materials and geometries, as demonstrated in Fig.4: quartz, glass substrates with plane and curved geometries. Moreover, consecutive transfer of monolayers on the same substrate allows creating 3D colloidal structures with controlled number of layers: sample in Fig.4d consists of 5 colloidal layers on its right side (the silica nanoparticle diameter is $580 \mathrm{~nm}$ in this case). The length of transferred colloidal stripes here was specially chosen to be different to reveal the high quality of every transferred layer. It should be noted 
a)

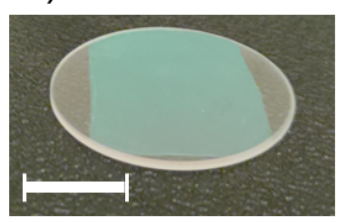

d) b)

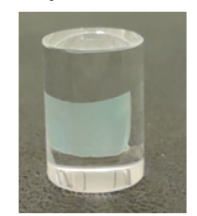

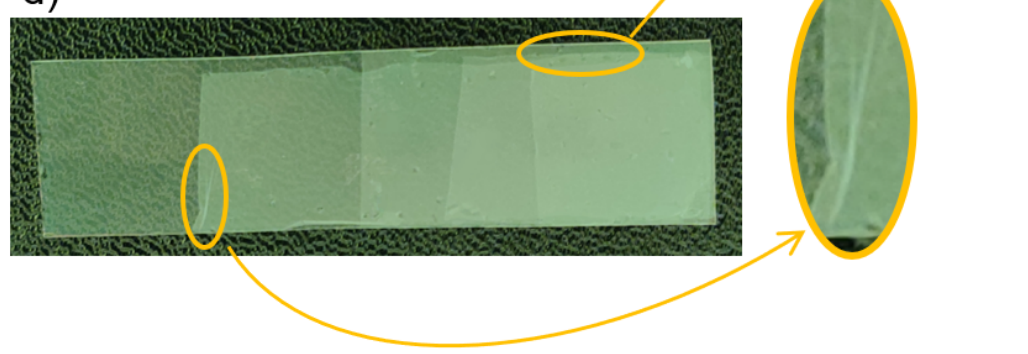

c)

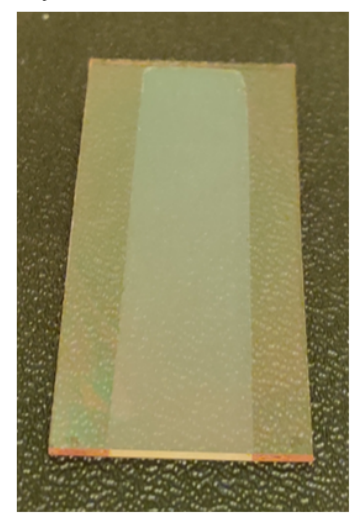

Figure 4. Photographs of colloidal crystals transferred on different target substrates: a) monolayer on quartz; b) monolayer on BK7 cylinder; c) monolayer on BK7 glass slide, covered with S1805 photoresist; d) from 1 (on the right) to 5 (on the left) monolayers on a polycarbonate susbtrate. Scale bar in a) is the same for all photographs and denotes $1 \mathrm{~cm}$. The diameter of colloidal particles used for the transfer is $580 \mathrm{~nm}$.

that in all samples in Figs.2 and Figs. 4 the inner parts of colloidal layers are practically free of defects visible by naked eye. The mechanical defects are concentrated mostly on the layers edge and can be of two types, as shown in Fig.4d insets: the layer rupture because of air bubbles trapped between the substrate and colloidal film (upper inset) and colloidal film folds which appear when the edge of a colloidal film detaches from the substrate during the water pumping out of the tank (lower inset). The both types of defects are avoidable with further improvements of the transfer process routine.

Figures 5b-f show the SEM photographs of the top surface of the sample from Fig.4d (the same as in Fig.5a) at regions with 1-5 transferred colloidal monolayers, respectively. The quality of hexagonal polycrystalline packaging does not depend on the layer's number and is possible due to the mechanical support of the thin parylene film.

\section{Nanopore arrays fabricated via NPL}

Due to the moderate experimental conditions under which the proposed transfer method occurs (room temperature, independence from the room illumination, water-assisted transfer), functional substrates conserve their properties. Consequently, substrates with transferred colloidal crystals can be used in photonic devices or be integrated into advanced experimental manipulations. Here we use the Nanosphere Photolithography (NPL) as an example of such an advanced fabrication procedure.

Figure 6a shows the same two samples as in Fig.2f with only one colloidal layer (nanoparticle diameter $580 \mathrm{~nm}$ ). Every sample has separate diffractive areas (8 areas 


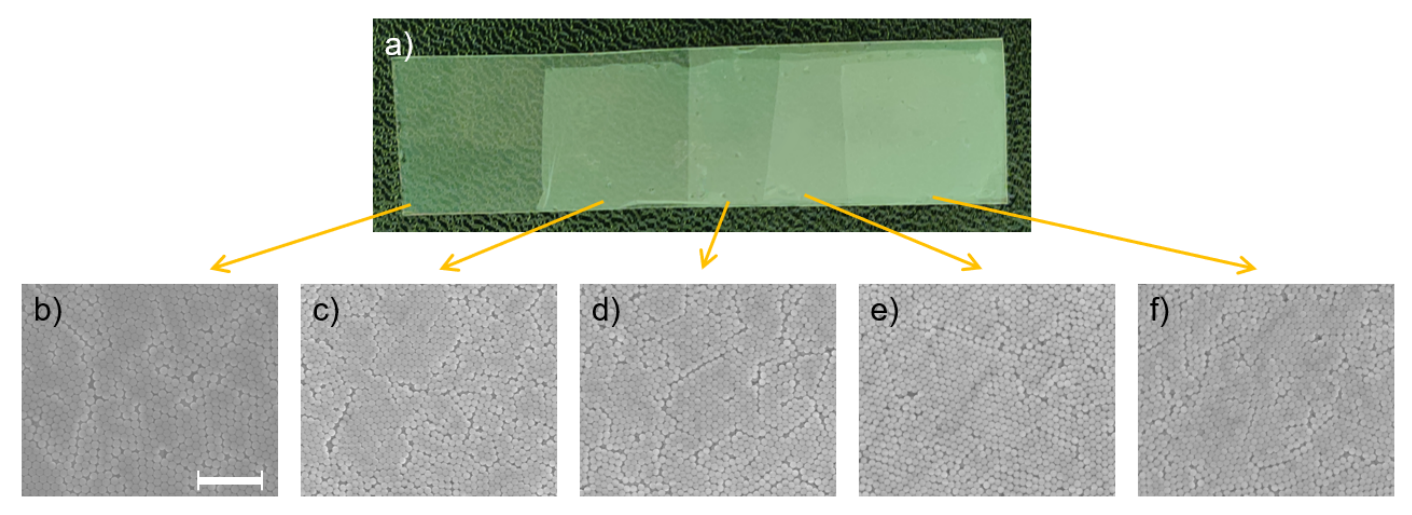

Figure 5. a) Photograph of the sample with up to 5 colloidal monolayers (the same as in Fig.4d); b)-f) SEM photographs of sample surface at regions with 1-5 transferred monolayers, respectively. Scale bar in b) is the same for all photographs b)-f) and denotes $5 \mu \mathrm{m}$. The diameter of colloidal particles is $580 \mathrm{~nm}$.

a)

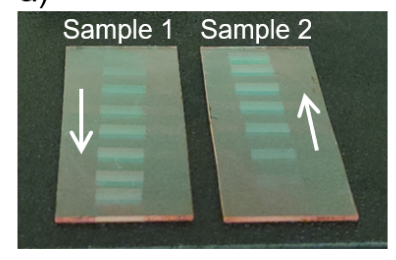

b)
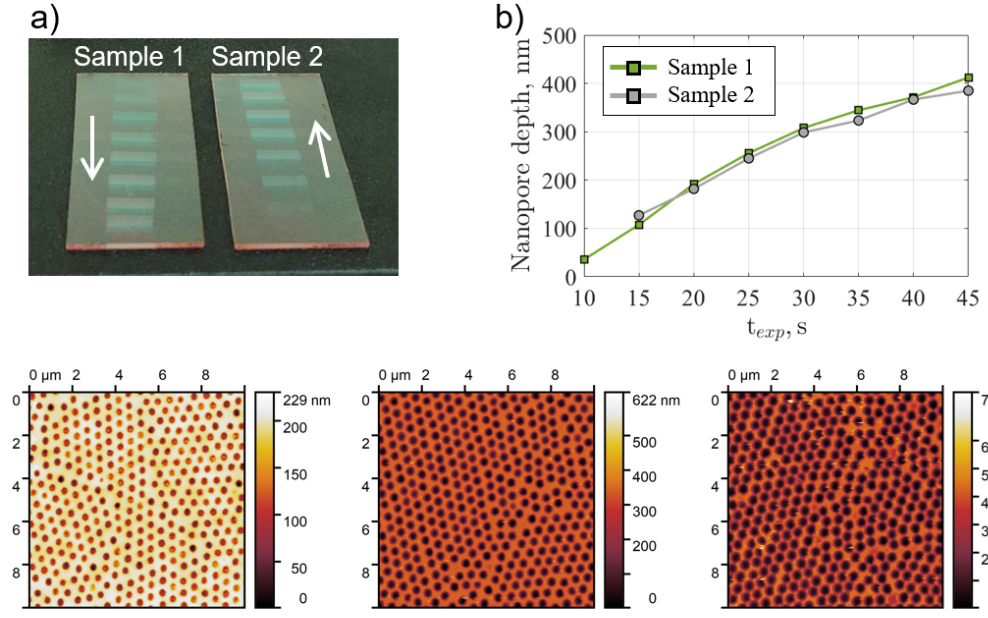

c)

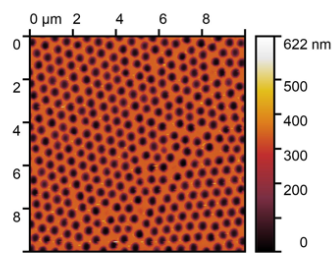

d)

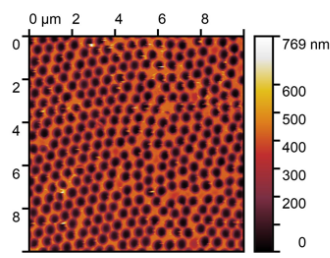

e)
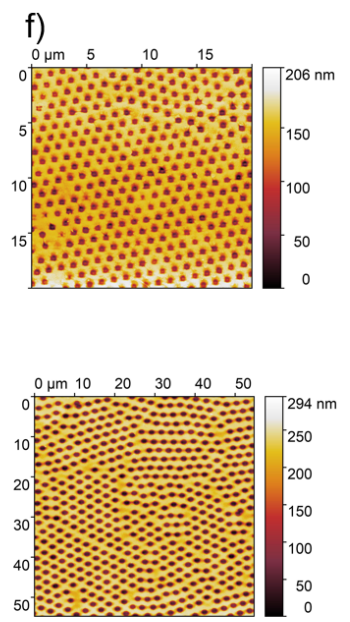

g)

Figure 6. a) Photograph of two samples with nanopore arrays, fabricated via NPL from transferred colloidal monolayers (the same as in Fig.2f). White arrows indicate the directions of nanopore depth growth. The diameter of initially transferred colloidal particles was $580 \mathrm{~nm}$; b) AFM measurements of nanopore depths in every UV-exposed area of Samples 1 and 2. Every area was exposed during the time $t_{\text {exp }} ; \mathrm{c}$ ), d) and e) AFM-measured topographies of Sample 2 areas with $t_{\text {exp }}=15 \mathrm{~s}, 30 \mathrm{~s}$ and $45 \mathrm{~s}$, correspondingly; f) and g) AFM-measured topographies of NPL-fabricated nanopore arrays, obtained from the transfer of colloidal particles of diameters $1100 \mathrm{~nm}$ and $2500 \mathrm{~nm}$, respectively. Nanopore depths for f) and g) are $116 \mathrm{~nm}$ and $200 \mathrm{~nm}$, respectively.

for Sample 1 and 7 areas for Sample 2), which were nanotextured via NPL with different UV-exposure times $t_{\text {exp }}$, and which consist of hexagonally arranged nanopores. The development time for all areas of both samples is the same $t_{d e v}=4 \mathrm{~s}$. The nanopore depth increases with $t_{\text {exp }}$, white arrows in Fig.6a indicate directions of the nanopore depth growth. The experimentally measured dependence of grating depth over the exposure time $t_{\text {exp }}$ is shown in Fig.6b for Samples 1 and 2. The fact that both curves 
almost coincide with each other proves the repeatability of results. The AFM-measured topographies of nanopore arrays are shown in Figs.6c-g (Fig.6f for nanoparticle diameter $1100 \mathrm{~nm}$, Fig.6g for $2500 \mathrm{~nm}$, other images for $580 \mathrm{~nm}$ ), they repeat the polycrystalline arrangement of initially transferred colloidal monolayers.

\section{Experimental measurements of EOT}

We utilized NPL to demonstrate the processing possibilities of the proposed colloidal transfer technology. Due to the NPL-compatibility the transferred thin films can be employed in active photonics [27], surface texturing [28], plasmonic sensors [29] etc. In this section we demonstrate that resonant plasmonic excitations are possible in metallized nanopore arrays, prepared via NPL and colloidal transfer, despite the disordered polycrystalline nature of samples. These results are consistent with our previous theoretical and experimental studies [26, 34].

For experiments we chose a sample analogous to one from Fig.4c with NPLfabricated array of nanopores from previously transferred close-packed colloidal particles with diameters of $580 \mathrm{~nm}$. The NPL exposure time is $t_{\text {exp }}=10 \mathrm{~s}$, resist development time is $t_{d e v}=4 \mathrm{~s}$, and AFM-measured nanopore depth is $40 \mathrm{~nm}$. The resist layer thickness is $600 \mathrm{~nm}$. In order to get a plasmonic structure the nanotextured resist surface was metallized with a $50 \mathrm{~nm}$-thin gold film using a thermal evaporation in vacuum, and the second $600 \mathrm{~nm}$-thin resist layer was spin-coated on the top. Thus, the symmetrical "Insulator-Metal-Insulator" (IMI) geometry was achieved, which supports the plasmonic Extraordinary Optical Transmission (EOT) effect [26, 35].

Figures 7a,e show the measured transmission spectra of the prepared structure in $\mathrm{TM}$ and TE polarizations, respectively, for incidence angles $0^{\circ}, 5^{\circ}$ and $10^{\circ}$. At normal incidence both spectra in Figs.7a,e demonstrate almost identical plasmonic peaks at $\lambda_{2} \approx 840 \mathrm{~nm}$; this resonant wavelength for the diffraction order $(m, n)$ can be estimated by the equation

$$
\lambda_{E O T}=\frac{\sigma_{0}}{\sqrt{\frac{4}{3}\left(m^{2}+m n+n^{2}\right)}} \sqrt{\frac{\varepsilon_{d} \cdot \operatorname{Re}\left(\varepsilon_{m}\right)}{\varepsilon_{d}+\operatorname{Re}\left(\varepsilon_{m}\right)}}
$$

for the hexagonal lattice $[36,26] ; \varepsilon_{d}$ and $\varepsilon_{m}$ are dielectric permittivities of dielectric and metallic layers, respectively, and $\sigma_{0}$ is the interpore distance defined by the diameter of the self-assembled particles. Taking $\varepsilon_{d} \approx 1.6^{2}$ for the S1805 dielectric resist, $\varepsilon_{m} \approx$ $(0.16+5.3 i)^{2}$ for gold and $\sigma_{0}=580 \mathrm{~nm}$, we estimate the EOT wavelength $\lambda_{E O T} \approx 840 \mathrm{~nm}$ defined by 6 symmetrical first diffraction orders $(m, n)=( \pm 1,0),(0, \pm 1),( \pm 1, \mp 1)$. It is worth mentioning that, in contrast to ideally periodical $2 \mathrm{D}$ hexagonal lattices with 6 discrete first diffraction orders (Fourier harmonics), the studied polycrystalline structure possesses a blurred diffraction ring because of randomly oriented nanopore crystallites of limited size [26].

With the increase of incidence angles the EOT spectral behavior becomes different in TE and TM: the EOT peak splits in TM, but stays at the same position $\lambda_{2}$ in TE. It 
can be explained using the Ewald's sphere approach in relation to plasmonic excitations, described in detail, for example, in [37].

Figures 7b-d explain the appearance of two EOT peaks for TM polarization in polycrystalline disordered structure at wavelengths $\lambda_{1}$ and $\lambda_{3}$ by multiple intersections of the plasmonic circle (denoted in black) with a blurred grating-imposed first order diffraction ring (denoted in green). These multiple intersections define multiple surface plasmon propagation directions, which merge into broad sectors (defined in brown). The width of sectors increases with the decrease of nanopore grain size. Thus, at inclined incidence there are two ways for plasmonic excitations in TM, shown in Figs.7b,d, and consequently two broad EOT peaks at $\lambda_{1}$ and $\lambda_{3}$.

The coupling of TE-polarized light to plasmons at $\lambda_{1}$ and $\lambda_{3}$ is suppressed, because propagation sectors are almost perpendicular to the light polarization at these wavelengths. However, the coupling is still possible at $\lambda_{2}$, as shown in Fig.7g.

a)

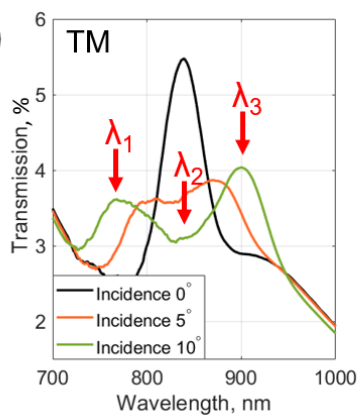

e)

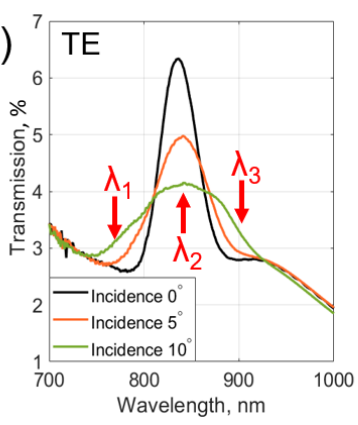

b) $\lambda_{1}=765 \mathrm{~nm}$

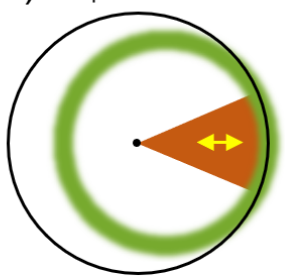

f)

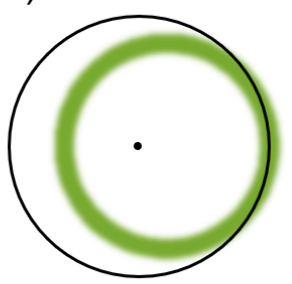

c) $\lambda_{2}=840 \mathrm{~nm}$

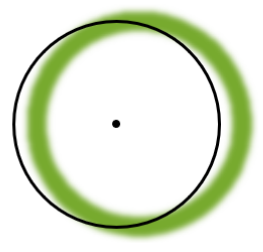

g)

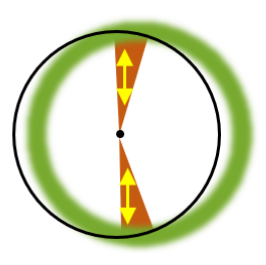

d) $\lambda_{3}=900 \mathrm{~nm}$

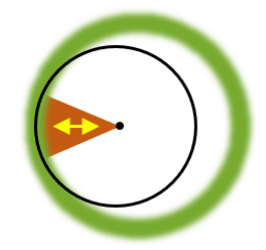

h)

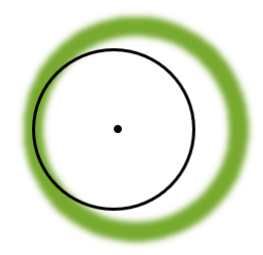

Figure 7. Extraordinary optical transmission through polycrystalline arrays of nanopores, fabricated via NPL and colloidal crystal transfer. a) Transmission in TM polarization; b)-d) diagrams in reciprocal space for different incident wavelengths denoted above each diagram. Black circles are plasmonic spheres with plasmon resonant wavevectors, green circles show first Fourier harmonics of polycrystalline 2D gratings (first diffraction orders). Colored brown sectors inside plasmonic spheres denote sets of wavevectors at which plasmonic resonances occur. Yellow arrows inside colored sectors demonstrate the incident light polarization; e) Transmission in TE polarization; f)-h) the diagrams similar to ones in b)-d), for plasmonic excitations at TE polarization.

The clear experimental observation of EOT in samples, fabricated via the proposed colloidal transfer method, confirms the preservation of diffraction structure quality 
during this transfer and demonstrates the possibility of its integration into multi-step fabrication processes.

\section{Conclusions}

In conclusion, we proposed a novel versatile colloidal crystal transfer technique compatible with a wide range of substrates regardless of their size, material, and wettability. There are no inherent limitations of colloidal particles material and size, too. In order to demonstrate the method possibilities we performed the colloidal transfer on quartz, glass substrates with a flat and curved surface, and prepared 3D colloidal structure with 5 overlaid monolayers. In addition, the process occurs at a room temperature in water and is independent from the illumination conditions. Consequently, it does not damage sensitive functional substrates and therefore can be implemented into more sophisticated fabrication chains.

As an example of functional substrates the photoresist S1805 was chosen; we managed to use the transferred colloidal monolayer as a mask for nanosphere photolithography. The metallized hexagonal arrays of nanopores demonstrated a clear extraordinary optical transmission behavior.

We believe that due to its high integration possibilities the proposed transfer technique will find applications in large-area surface nanotexturing, plasmonics, and will speed up a device fabrication process.

\section{Acknowledgements}

The work was funded by the SIS 488 doctoral school of Saint-Etienne, university of Lyon (France).

The authors would like to thank UJM engineer Frédéric Celle for the technical support and CEA Ing. de Recherche M. Philippe Berne for his assistance and fruitful discussions.

\section{Data availability statement}

All data that support the findings of this study are included within the article (and any supplementary files).

\section{ORCID iDs}

Andrei A. Ushkov https://orcid.org/0000-0001-8962-1599

Yves Jourlin https://orcid.org/0000-0002-7935-2150

Olivier Lebaigue https://orcid.org/0000-0001-6676-9216 


\section{References}

[1] Cai, Z., Li, Z., Ravaine, S., He, M., Song, Y., Yin, Y., Zheng, H., Teng, J., Zhang, A.: From colloidal particles to photonic crystals: advances in self-assembly and their emerging applications. Chemical Society Reviews (2021)

[2] Zhao, Y., Shang, L., Cheng, Y., Gu, Z.: Spherical colloidal photonic crystals. Accounts of chemical research 47(12), 3632-3642 (2014)

[3] Li, H., He, F., Ji, C., Zhu, W., Xu, Y., Zhang, W., Meng, X., Fang, X., Ding, T.: Purcell-enhanced spontaneous emission from perovskite quantum dots coupled to plasmonic crystal. The Journal of Physical Chemistry C 123(41), 25359-25365 (2019)

[4] Zhang, J., Zhu, Z., Yu, Z., Ling, L., Wang, C.-F., Chen, S.: Large-scale colloidal films with robust structural colors. Materials Horizons 6(1), 90-96 (2019)

[5] Liu, P., Bai, L., Yang, J., Gu, H., Zhong, Q., Xie, Z., Gu, Z.: Self-assembled colloidal arrays for structural color. Nanoscale Advances 1(5), 1672-1685 (2019)

[6] Chan, L., Ghoshal, A., DeCuir Jr, E.A., Chen, Y.P., Morse, D.E., Gordon, M.J.: Fabrication and optical behavior of graded-index, moth-eye antireflective structures in CdTe. Journal of Vacuum Science \& Technology B, Nanotechnology and Microelectronics: Materials, Processing, Measurement, and Phenomena 35(1), 011201 (2017)

[7] Lora Gonzalez, F., Chan, L., Berry, A., Morse, D.E., Gordon, M.J.: Simple colloidal lithography method to fabricate large-area moth-eye antireflective structures on $\mathrm{Si}$, Ge, and GaAs for IR applications. Journal of Vacuum Science \& Technology B, Nanotechnology and Microelectronics: Materials, Processing, Measurement, and Phenomena 32(5), 051213 (2014)

[8] Chan, L.W., Morse, D.E., Gordon, M.J.: Moth eye-inspired anti-reflective surfaces for improved ir optical systems \& visible leds fabricated with colloidal lithography and etching. Bioinspiration \& biomimetics 13(4), 041001 (2018)

[9] Karg, M., König, T.A., Retsch, M., Stelling, C., Reichstein, P.M., Honold, T., Thelakkat, M., Fery, A.: Colloidal self-assembly concepts for light management in photovoltaics. Materials Today 18(4), 185-205 (2015)

[10] Qi, Q., Liu, C., Liu, L., Meng, Q., Wei, S., Ming, A., Zhang, J., Wang, Y., Wu, L., Zhu, X., Wei, F., Yan, J.: Fabrication, characterization, and application of large-scale uniformly hybrid nanoparticle-enhanced raman spectroscopy substrates. Micromachines 10(5), 282 (2019)

[11] Sarkar, S., Gupta, V., Kumar, M., Schubert, J., Probst, P.T., Joseph, J., König, T.A.: Hybridized guided-mode resonances via colloidal plasmonic self-assembled grating. ACS applied materials \& interfaces 11(14), 13752-13760 (2019)

[12] Khanna, S., Utsav, Marathey, P., Chaliyawala, H., Rajaram, N., Roy, D., Banerjee, R., Mukhopadhyay, I.: Fabrication of long-ranged close-packed monolayer of silica nanospheres by spin coating. Colloids and Surfaces A: Physicochemical and Engineering Aspects 553, 520-527 (2018)

[13] Garcia Nunez, C., Navaraj, W.T., Liu, F., Shakthivel, D., Dahiya, R.: Large-area self-assembly of silica microspheres/nanospheres by temperature-assisted dip-coating. ACS applied materials \& interfaces 10(3), 3058-3068 (2018)

[14] Tran, G.T., Koike, M., Uchikoshi, T., Fudouzi, H.: Fabrication of polystyrene colloidal crystal film by electrophoretic deposition. Advanced Powder Technology 31(8), 3085-3092 (2020)

[15] Reculusa, S., Ravaine, S.: Synthesis of colloidal crystals of controllable thickness through the Langmuir-Blodgett technique. Chemistry of materials 15(2), 598-605 (2003)

[16] Denkov, N., Velev, O., Kralchevski, P., Ivanov, I., Yoshimura, H., Nagayama, K.: Mechanism of formation of two-dimensional crystals from latex particles on substrates. Langmuir 8(12), 3183-3190 (1992)

[17] Wu, H., Niu, G., Ren, W., Jiang, L., Liang, O., Zhao, J., Liu, Y., Xie, Y.-H.: Crucial impact of hydrophilicity on the self-assembled $2 \mathrm{~d}$ colloidal crystals using Langmuir-Blodgett method. Langmuir 36(34), 10061-10068 (2020) 
[18] Kazumasa, H., Tomohiro, S., Toyoak, H., Isao, T., Teruo, H.: Effect of surface properties of substrate on structure of closely packed colloidal crystal deposited on fiber surface through dip-coating. Journal of Textile Engineering 65(5) (2019)

[19] Yan, X., Yao, J., Lu, G., Chen, X., Zhang, K., Yang, B.: Microcontact printing of colloidal crystals. Journal of the American Chemical Society 126(34), 10510-10511 (2004)

[20] Lee, B.H., Shin, H., Sung, M.M.: Patterning a two-dimensional colloidal crystal by water-mediated particle transfer printing. Chemistry of Materials 19(23), 5553-5556 (2007)

[21] Li, W., Yang, B., Wang, D.: Fabrication of colloidal crystals with defined and complex structures via layer-by-layer transfer. Langmuir 24(23), 13772-13775 (2008)

[22] Mihi, A., Zhang, C., Braun, P.V.: Transfer of preformed three-dimensional photonic crystals onto dye-sensitized solar cells. Angewandte Chemie International Edition 50(25), 5712-5715 (2011)

[23] Kohoutek, T., Parchine, M., Bardosova, M., Fudouzi, H., Pemble, M.: Large-area flexible colloidal photonic crystal film stickers for light trapping applications. Optical Materials Express 8(4), 960-967 (2018)

[24] Banik, M., Bhandaru, N., Mukherjee, R.: Transfer printing of colloidal crystals based on uv mediated degradation of a polymer thin film. Chemical Communications 54(28), 3484-3487 (2018)

[25] Zhang, Z., Geng, C., Hao, Z., Wei, T., Yan, Q.: Recent advancement on micro-/nano-spherical lens photolithography based on monolayer colloidal crystals. Advances in colloid and interface science 228, 105-122 (2016)

[26] Ushkov, A., Dellea, O., Verrier, I., Kampfe, T., Shcherbakov, A., Michalon, J.-Y., Jourlin, Y.: Compensation of disorder for extraordinary optical transmission effect in nanopore arrays fabricated by nanosphere photolithography. Optics Express 28(25), 38049-38060 (2020)

[27] Szabó, Z., Volk, J., Fülöp, E., Deák, A., Bársony, I.: Regular ZnO nanopillar arrays by nanosphere photolithography. Photonics and Nanostructures-Fundamentals and Applications 11(1), 1-7 (2013)

[28] Geng, C., Yan, Q., Du, C., Dong, P., Zhang, L., Wei, T., Hao, Z., Wang, X., Shen, D.: Large-area and ordered sexfoil pore arrays by spherical-lens photolithography. ACS Photonics 1(8), 754-760 (2014)

[29] Chang, Y.-C., Chung, H.-C., Lu, S.-C., Guo, T.-F.: A large-scale sub-100 nm Au nanodisk array fabricated using nanospherical-lens lithography: a low-cost localized surface plasmon resonance sensor. Nanotechnology 24(9), 095302 (2013)

[30] Dellea, O., Lebaigue, O., Poncelet, O.: Process for Forming a Particle Film on the Surface of a Substrate. FR3095138B1, 2021

[31] Fortin, J., Lu, T.-M.: Ultraviolet radiation induced degradation of poly-para-xylylene (parylene) thin films. Thin Solid Films 397(1-2), 223-228 (2001)

[32] Jean, J., Wang, A., Bulović, V.: In situ vapor-deposited parylene substrates for ultra-thin, lightweight organic solar cells. Organic Electronics 31, 120-126 (2016)

[33] He, Q., Liu, J.-Q., Yang, B., Chen, X., Yang, C.-S.: Parylene c composite film modified by nanosilica/titania for enhancement of thermal stability and uv stability. Surface and Coatings Technology 252, 120-125 (2014)

[34] Ushkov, A., Dellea, O., Verrier, I., Kampfe, T., Shcherbakov, A., Michalon, J.-Y., Jourlin, Y.: Nanosphere photolithography: The influence of nanopore arrays disorder on extraordinary optical transmission. In: PHOTOPTICS, pp. 46-53 (2021)

[35] Ushkov, A.A., Shcherbakov, A.A., Verrier, I., Kampfe, T., Jourlin, Y.: Systematic study of resonant transmission effects in visible band using variable depth gratings. Scientific reports 9(1), 1-9 (2019)

[36] Ekşioğlu, Y., Cetin, A.E., Petráček, J.: Optical response of plasmonic nanohole arrays: comparison of square and hexagonal lattices. Plasmonics 11(3), 851-856 (2016)

[37] Sauvage-Vincent, J., Jourlin, Y., Tonchev, S., Veillas, C., Claude, P., Parriaux, O.: Industrial fabrication of an optical security device for document protection using plasmon resonant 
transmission through a thin corrugated metallic film embedded on a plastic foil. In: MicroOptics 2012, vol. 8428, p. 84280 (2012). International Society for Optics and Photonics 